\title{
Actitud hacia la Ciencia: desarrollo y validación estructural del School Science Attitude Questionnaire (SSAQ)
}

\author{
David Aguilera \\ Departamento de Didáctica de las Ciencias Experimentales. Universidad de Granada. España. \\ davidaguilera@ugr.es \\ ORCID: http:/ / orcid.org/0000-0002-7996-9097 \\ F. Javier Perales-Palacios \\ Departamento de Didáctica de las Ciencias Experimentales. Universidad de Granada. España. \\ fperales@ugr.es \\ ORCID: bttp:// orcid.org/0000-0002-6112-2779
}

[Recibido: 20 Noviembre 2018. Revisado: 12 Enero 2019. Aceptado: 10 Mayo 2019]

\begin{abstract}
Resumen: El objetivo de este estudio es facilitar la labor docente e investigadora en relación a la actitud hacia la ciencia del alumnado, mediante el desarrollo y la validación de la versión española del School Science Attitude Questionnaire (SSAQ). Tras una revisión de la literatura, pudimos observar la escasez de instrumentos diseñados y validados sobre dicha temática en idioma español, de este modo el SSAQ se ha constituido a partir de un instrumento de reciente creación, con validez de contenido y pilotado, pero carente de un soporte teórico sólido y de validez de constructo. El proceso que hemos seguido atiende a su traducción del inglés al español y a la idoneidad del contenido mediante valoración de expertos a fin de realizar la validación en el contexto académico español. La validez psicométrica del instrumento, constituido por cuatro dimensiones y 20 ítems, se realizó sobre una muestra de 259 alumnos de Educación Secundaria Obligatoria ( $1^{\circ}$ a $4^{\circ}$ curso). El análisis estructural exploratorio arroja unos valores satisfactorios, al igual que el confirmatorio, en el que el ajuste estadístico es adecuado según los valores obtenidos para $\chi^{2}$ g.l. (1.028), GFI (.986), RMR (.056) y NFI (.980). Finalmente, las cuatro dimensiones y los ítems que componen la estructura del SSAQ se ajustan al vínculo existente entre creencia y actitud según la Teoría de la Acción Razonada.
\end{abstract}

Palabras clave: Enseñanza de las ciencias; actitud hacia la ciencia; validez de constructo; cuestionario.

Attitude towards Science: Development and structural validation of School Science Attitude Questionnaire (SSAQ)

Abstract: The objective of this study is to facilitate teaching and research related to students' attitude toward science, by development and validation of Spanish version of the School Science Attitude Questionnaire (SSAQ). Following a thorough review of the literature, we could observe the shortage of instruments designed and validated in Spanish, so the SSAQ has been constructed from a recently created instrument, with content validity and piloted, but lacking of a solid theoretical support and construct validity. The process followed attends to its translation from English into Spanish and suitability of the content through expert assessment in order to carry out the validation in the Spanish academic context. The psychometric validity of the instrument, consisting of four factors and 20 items, was performed on a sample of 259 students of Secondary Education (1st to 4th grade). The exploratory structural analysis yields satisfactory values, also the confirmatory factor analysis shows an adequate statistical fit, according to the values obtained for $\chi^{2} /$ d.f. (1.028), CFI (.986), RMR (.056) and NFI (.980). Finally, the four factors and the items that make up the structure of the SSAQ are adjusted to the link between belief and attitude according to the Theory of Reasoned Action.

Keywords: Science education; attitude towards science; construct validity; questionnaire.

Para citar este artículo: Aguilera D., Perales-Palacios F.J. (2019) Actitud hacia la Ciencia: Desarrollo y validación estructural del School Science Attitude Questionnaire (SSAQ). Revista Eureka sobre Enseñanza y Divulgación de las Ciencias 16(3), 3103. doi: 10.25267/Rev_Eureka_ensen_divulg_cienc.2019.v16.i3.3103 


\section{Introducción}

La evaluación de la actitud hacia la ciencia (AC) del alumnado se ha abordado en numerosos trabajos, generando una producción científica considerable (Osborne, Simon y Collins 2003). No obstante, valorar la actitud de un individuo no es una tarea sencilla, dado que son múltiples los factores que actúan como moderadores, provocando que la actitud de un individuo oscile en el rango mala-excelente (Schwarz 2007). En este sentido, Stark y Gray (1999) afirman que el desencanto mostrado por los estudiantes hacia la ciencia escolar podría deberse a las actividades, explicaciones u otras acciones que acontecen durante las clases de ciencias, pudiendo no ser de su agrado. Al hilo de este acontecimiento, Mazas y Bravo-Torija (2018) recogen en su estudio que un porcentaje elevado de los futuros maestros y maestras de Educación Infantil y Primaria reconocen no tener recursos y conocimientos suficientes para enseñar ciencias.

La situación descrita nos invita a revisar aquellos instrumentos diseñados y utilizados para evaluar el constructo de AC con el fin de proveer de instrumentos fiables y válidos, no sólo a aquellos que investigan en el área de Didáctica de las Ciencias Experimentales, sino también a maestros y profesores de ciencias con objeto de facilitar su labor docente. En este sentido, Summers y Abd-El-Khalick (2018), además de aportar el proceso de desarrollo y validación de un nuevo cuestionario (BRAINS) para medir la AC del alumnado, revisan el estado de desarrollo y validación de otros 17 instrumentos ya existentes.

A estos instrumentos, todos ellos en inglés, hemos de añadir aquellos creados (o adaptados) y validados en español, pues son estos últimos los que marcarán la necesidad de incrementar o no el número de cuestionarios en dicho idioma para medir el constructo AC. Así, nos encontramos ante dos instrumentos en español que aportan datos acerca de su fiabilidad y validez de constructo, estos son: los cuestionarios TOSRA para hispano hablantes (Navarro, Förster, González y González-Pose 2016) y S-SSAS (Toma y Meneses-Villagrá 2019). El primero consta de 70 ítems y está destinado a Educación Secundaria, mientras que el segundo se compone de 10 ítems y fue diseñado para alumnado de Educación Primaria. También han de considerarse los cuestionarios PAC, para estudiantes de Educación Secundaria, Bachillerato y Universidad (Vázquez y Manassero 1997), ROSE, destinado a alumnado de los últimos cursos de Educación Primaria y Educación Secundaria, (p. ej.: Marbá-Tallada y Márquez 2010; Vázquez y Manassero 2008) y PANA, para alumnado de Educación Primaria y Secundaria (Pérez-Manzano 2012). Estos últimos tres cuestionarios han sido traducidos o diseñados directamente en idioma español, pero no han probado su validez y fiabilidad (p. ej.: PANA) o no muestran una validez y fiabilidad sólidas (p. ej.: la versión en español de ROSE y PAC).

A la luz de la revisión de la literatura comentada, se deduce que disponemos de escasos instrumentos con fiabilidad y validez contrastada para medir la AC del alumnado hispano hablante. Ello acentúa la necesidad de desarrollar, traducir o adaptar nuevos instrumentos capaces de medir un constructo tan complejo y multifacético como es la AC. En este sentido, buscando aportar un instrumento más a la limitada gama de cuestionarios disponibles para valorar la AC del alumnado, el presente trabajo pretende:

(1) Traducir al español y adaptar al constructo AC, el cuestionario de Barak, Ashar y Dori (2011) basado en el SMQ (Glynn y Koballa 2006).

(2) Realizar una validación estructural exploratoria de la versión traducida para la etapa de Educación Secundaria Obligatoria (ESO). 


\section{Contextualización del School Science Attitude Questionnaire}

El instrumento, sobre el cual versa este trabajo, surge a partir de una exhaustiva revisión de la literatura y de las intenciones descritas anteriormente. Así, dicha revisión nos permitió identificar un cuestionario basado en el SMQ (Glynn y Koballa 2006) diseñado por los autores Barak et al. (2011), los cuales determinaron su validez de contenido (evaluación por expertos y maestros del área de Didáctica de las Ciencias Experimentales) y aportaron datos sobre su fiabilidad (Alfa de Cronbach $=.88$ ). Sin embargo, las modificaciones que Barak et al. (2011) realizaron para crear el nuevo instrumento fueron sustanciales:

- Redujeron el número de ítems, pasando de 30 a 20.

- Reformularon los ítems para adaptarlos a las características del alumnado de Educación Primaria.

- Transformaron la escala tipo Likert de 1 a 5 del instrumento original (nunca-siempre), basada en frecuencias, por una de orientación evaluativa (muy en desacuerdo-muy de acuerdo).

- Crearon unas dimensiones nuevas, diferentes a las del cuestionario original.

Como consecuencia de lo anterior, Barak et al. (2011) acercaron su cuestionario más al constructo «actitud hacia la ciencia» que al de «motivación hacia la ciencia» (los siguientes apartados inciden sobre ello). A la vez los cambios descritos dan lugar a un instrumento diferente, hecho que obliga a aportar un soporte teórico claro y consistente (Messick 1989) en aras de ejecutar una validación de constructo e identificar una fiabilidad robusta (Pearl 1974).

En definitiva, dado que el SMQ fue el instrumento que inspiró a Barak et al. (2011) para confeccionar el anteriormente descrito y considerando el constructo al que este se pretende adaptar (AC), decidimos denominar dicho instrumento como «School Science Attitude Questionnaire (SSAQ)».

\section{El concepto actitud, actitud hacia la ciencia y sus moderadores}

El concepto de actitud y su interpretación ha evolucionado desde su aparición hasta los modelos teóricos aceptados actualmente. Así, la influencia ejercida tradicionalmente por la psicología social y educativa en la definición del concepto AC modificó la consideración conductista por la cognitivista (Richardson 1996), para finalmente situar el concepto de actitud dentro del dominio afectivo, hecho que llevó a definir la AC como la disposición y la inclinación afectiva positiva o negativa por aprender ciencia (Koballa y Glynn 2007). Sin embargo, las actitudes no aparecen de forma aislada, sino que se encuentran estrechamente relacionadas con factores cognitivos y conductuales, además del ya citado afectivo. Ello se ha explicado satisfactoriamente en la «Teoría de la Acción Razonada» (Fishbein y Ajzen 1975), convirtiéndose en una de las más referenciadas para argumentar el concepto de AC.

La «Teoría de la Acción Razonada» aborda los constructos creencia, actitud, intención de comportamiento y comportamiento, los cuales se vinculan entre sí, no significando esto que sean constructos idénticos. Por tanto, mientras la actitud se refiere a la evaluación favorable y desfavorable que realiza el individuo sobre algo, las creencias representan la información que posee este sobre dicho elemento (Fishbein y Ajzen 1975). Tan es así, que la actitud es un constructo de dominio afectivo, con elevada carga evaluativa y determinada por las creencias (Ajzen y Fishbein 2005). En resumen, las creencias que posee un individuo hacia algo derivan en un conjunto de reacciones afectivas (favorables o desfavorables) hacia el mismo (actitud), las cuales predisponen a dicho individuo a ejecutar un comportamiento concreto (Ramsden 1998). No obstante, Koballa (1988a) puntualiza que la intención de comportamiento no determina completamente el comportamiento de un individuo. Siguiendo la teoría descrita, la 
valoración de la actitud del alumnado hacia la ciencia debería centrar su atención, por un lado, en las creencias y percepciones relativas a la capacidad/habilidad científica, importancia y utilidad de la ciencia, y por otro, a las reacciones afectivas que aquel manifiesta en forma de interés y disfrute hacia el estudio de la ciencia. Así, todos los componentes descritos podrían constituir un medio adecuado para medir la AC del alumnado (Speering y Rennie 1996).

Los supuestos teóricos que respaldan al concepto de actitud y, en consecuencia, al de AC, resaltan la complejidad y multidimensionalidad de este constructo y, con ello, la posibilidad de que numerosos factores pudieran influir en aquella (Newell, Tharp, Moreno, Zientek y Vogt 2015). En este sentido, las variables moderadoras más estudiadas han sido el sexo y la edad del alumnado (Potvin y Hasni 2014).

En cuanto al sexo del alumnado, se trata de una de las variables moderadoras que más resultados contradictorios parece haber generado en esta línea de investigación, puesto que algunos estudios afirman que los niños suelen mostrar mejor AC que las niñas (Denessen, Vos, Hasselman y Louws 2015; De Witt y Archer 2015; Pro y Pérez-Manzano 2014), mientras que otros no observan diferencia alguna (Akpinar, Yildiz, Tatar y Ergin 2009; Toma y Greca 2018). Por ende, parece que todavía se está lejos de alcanzar un consenso acerca de la relación entre el sexo del alumnado y su AC. Por el contrario, los análisis realizados por múltiples estudios parecen consensuar que la edad, asociada a la promoción de los estudiantes en las distintas etapas del sistema educativo, es una variable que incide también en la AC. Esta relación podría describirse como inversa, pues a medida que el alumnado avanza de curso, las actitudes favorables hacia la ciencia disminuyen drásticamente (Ali, Yager, Hacieminoglu y Caliskan 2013; Marbá-Tallada y Márquez 2010; Said, Summers, Abd-El-Khalick y Wang 2016; Vázquez y Manassero 2008). En esta misma línea de variables intrínsecas, el rendimiento académico constituye otra variable de recurrente consideración, estando altamente correlacionado un buen rendimiento académico en las asignaturas de naturaleza científica con una buena AC (Caleon y Subramaniam 2008). También la competencia científica del alumnado, así como la dificultad que este percibe sobre el aprendizaje de los contenidos científicos, parecen estar relacionados con la actitud que este muestra hacia el aprendizaje de las ciencias (Chi, Wang, Liu y Zhu 2017).

Por otra parte, diferentes estudios han centrado su atención en aquellas variables extrínsecas al alumno, de forma que se han aportado datos que identifican la influencia en la AC del alumnado de: el método de enseñanza empleado (Aguilera y Perales 2018a), determinados recursos didácticos como el libro de texto o las ilustraciones (Aguilera y Perales 2018b), el nivel de desarrollo del país en el que reside el alumno (Sjøberg y Schreiner 2010) o la ocupación de sus padres (Chi et al. 2017).

\section{Método}

Este trabajo presenta la validación de constructo del SSAQ, la cual se ha realizado conforme a las directrices de Lloret-Segura, Ferreres-Traver, Hernández-Baeza y Tomás-Marco (2014). Así, se ha desarrollado un AFE (Análisis Factorial Exploratorio), al que le ha seguido un análisis factorial confirmatorio (AFC).

\section{Participantes}

La muestra la conforman 259 estudiantes de ESO, la selección de estos se realizó mediante muestreo no probabilístico intencional por razones de accesibilidad. La conveniencia de la muestra radicó en la participación del centro educativo en un proyecto de investigación e innovación en colaboración con la Universidad de Granada (a través de los autores de este trabajo). Los datos proceden del alumnado matriculado en los cursos de $1^{\circ}$ a $4^{\circ}$ de ESO en un 
instituto del sur de Córdoba (España), participando un total de 130 alumnos y 129 alumnas (Tabla 1). A excepción de 25 de cuarto curso, que cursaban los itinerarios de Ciencias Sociales y Humanidades, el resto cursaban asignaturas de ciencias.

Tabla 1. Datos socio-demográficos de la muestra.

\begin{tabular}{cccccc}
\hline Sexo & $\mathbf{1}^{\mathbf{0}} \mathbf{E S O}$ & $\mathbf{2}^{\mathbf{o}} \mathbf{E S O}$ & $\mathbf{3}^{\mathbf{0}}$ ESO & $\mathbf{4}^{\mathbf{0}}$ ESO & Total \\
\hline Hombre & 29 & 24 & 42 & 35 & 130 \\
\hline Mujer & 28 & 30 & 43 & 28 & 129 \\
\hline Total & 57 & 54 & 85 & 63 & 259 \\
\hline
\end{tabular}

La participación de los estudiantes ha sido autorizada por la Administración educativa correspondiente, en este caso la Consejería de Educación de la Junta de Andalucía; además ha sido aprobada en el Consejo escolar del centro educativo y por los representantes legales de cada alumno.

\section{Traducción del instrumento}

La traducción del cuestionario se realizó según el procedimiento sugerido por Borsa, Damásio y Bandeira (2012). Así, en primer lugar un maestro bilingüe tradujo el cuestionario del inglés al español. Posteriormente, una maestra (también bilingüe) llevó a cabo una traducción inversa al inglés de la versión española. Para finalizar, se comparó la versión original con la traducción inversa, observando una alta correspondencia. Ello se evidenció en la estructura y el significado de cada uno de los ítems, resultando semejantes la versión inglesa original y la traducción inversa. A modo de ejemplo, el ítem 1 «I think that science is an interesting subject» (versión original) mantuvo su estructura y significado en la traducción inversa «I think that science is an interesting topic», pues la variante resultó equivalente al tratarse de sinónimos.

\section{Adaptación del instrumento}

La relación entre actitud y motivación es evidente, de ahí que a menudo se usen ambos términos como sinónimos. No obstante, actitud y motivación son dos constructos diferentes con referentes teóricos propios (Koballa y Glynn 2007). Al hilo de esta situación, decidimos adaptar el cuestionario diseñado por Barak et al. (2011) al constructo teórico más próximo, correspondiéndose al de $\mathrm{AC}$ según sus características, en lugar de la motivación hacia el aprendizaje de esta (constructo medido por el SMQ, en el cual se basa). Ello se debe principalmente a dos cuestiones:

(1) La amplia mayoría de los ítems se expresan en términos afectivos y/o de creencia (solamente los ítems 15 y 16 se expresan en términos de acción), hecho que lo hace afín al constructo de actitud según la «Teoría de la Acción Razonada» (Fishbein y Ajzen 1975).

(2) Las dimensiones que lo componen (importancia de la ciencia para el estudiante; autoeficacia; interés y disfrute; y conexión de la ciencia con la vida diaria del estudiante) lo aproximan al constructo AC, sustentándose esto en el estudio desarrollado por Speering y Rennie (1996), quienes afirman que aquella se constituye a partir de:

- la percepción del estudiante sobre su rendimiento en ciencias (autoeficacia);

- las expectativas de futuro en el estudio de las ciencias (conexión con la vida diaria);

- la importancia y utilidad de la ciencia percibida por el estudiante (importancia de la ciencia);

- y el disfrute con su práctica o estudio (interés y disfrute). 
Por el contrario, lo que reafirma la necesidad de esta adaptación, observamos que Glynn y Koballa (2006) describen cinco dimensiones para medir la motivación del alumnado por aprender ciencia netamente diferentes a las propuestas en Barak et al. (2011):

- motivación intrínseca;

- motivación extrínseca;

- autodeterminación;

- autoeficacia;

- y ansiedad provocada por la evaluación.

Por estas razones, el cuestionario creado por Barak et al. (2011), al cual hemos nombrado SSAQ, se ajusta en mayor medida al concepto AC, alejándose considerablemente del constructo teórico medido por el SMQ, es decir, la motivación hacia el aprendizaje de la ciencia.

Tras determinar el constructo medido por el SSAQ según las dimensiones y los ítems que lo componen, se procedió a analizar su ajuste a la definición de AC. Como hemos referido, según la «Teoría de la Acción Razonada» la actitud es una reacción afectiva (positiva o negativa) hacia algo, la ciencia en nuestro caso, la cual está supeditada por las creencias que pueda poseer el estudiante sobre esta (Fishbein y Ajzen 1975). Por tanto, parece coherente afirmar que si pretendemos evaluar la AC del alumnado debemos atender a sus creencias y afinidad afectiva hacia esta. Consecuentemente, y ajustándose a la definición de actitud dada por Koballa y Glynn (2007), los ítems de un cuestionario que pretende medir el constructo AC deben redactarse en términos de creencia (p. ej.: Creo/Pienso que...) o de afinidad afectiva (p. ej.: Me gusta.../Me divierte...). Así, a fin de ajustar el SSAQ a los fundamentos teóricos descritos, se modificó el ítem 16 redactado en términos de acción en su versión inicial («Leo artículos y veo programas de televisión que tratan temas científicos») y expresado en términos afectivos en la versión actual («Me gusta leer artículos y ver programas que tratan temas científicos»). El ítem 15 «Ayudo a los demás en las clases de ciencias », a pesar de estar redactado también en términos de acción, no fue modificado debido a las implicaciones de la acción de ayudar: cognitivas (evaluación de la capacidad/habilidad científica), afectivas (empatía) y conductuales (claro ejemplo de predisposición).

Para finalizar el proceso de adaptación, un doctor en Didáctica de las Ciencias Experimentales y dos docentes (un maestro de Educación Primaria y un profesor de ESO) con experiencia en la enseñanza de las ciencias, verificaron que los ítems fuesen apropiados para ser suministrados a alumnos de ESO.

\section{Formato del instrumento y definición de sus dimensiones}

Los 20 ítems que componen al SSAQ se distribuyen en cuatro factores latentes (dimensiones) no jerárquicos. De este modo el agrupamiento de los ítems es el siguiente:

- Importancia de la ciencia para el estudiante (D1): valora la relevancia otorgada por el alumnado a las asignaturas de ciencias, al conocimiento científico adquirido en ellas y, derivado de lo anterior, a la ciencia en general. De este modo, cuanto mayor sea la puntación en este factor, mayor será el valor dado por el estudiante a la ciencia y la ciencia escolar (ítems: 5*, 6, 9, 13, 18).

- Autoeficacia (D2): atiende a las creencias y percepciones del alumnado sobre sus capacidades y habilidades para tener éxito en el estudio de la ciencia. Por tanto, aquellos que obtengan altas puntuaciones en esta dimensión demuestran una confianza 
alta en sus capacidades y habilidades para comprender, aprender y hacer ciencia (ítems: $4,10,14,15,19 *)$.

- Interés y disfrute (D3): se refiere al nivel de interés y disfrute que el alumnado experimenta al aprender o hacer ciencia. Tan es así que puntuaciones altas significarán un nivel de interés y disfrute elevado hacia la práctica y el aprendizaje de los contenidos científicos (ítems: 1, 7, 8*,11,17).

- Conexión de la ciencia con la vida diaria del estudiante (D4): considera la utilidad de la ciencia percibida por el alumnado y sus deseos de realizar estudios superiores relacionados con esta, reflejándose todo ello en la vinculación de las ciencias con la vida cotidiana de aquel. Por ende, puntuar alto en este factor se interpreta como una vinculación estrecha de las ciencias con la vida diaria del estudiante (ítems: 2, 3, 12, 16, $20 *$.

Todos los ítems han sido redactados en positivo, exceptuando aquellos marcados con asterisco, los cuales se expresan en negativo (ver anexo) a fin de evitar respuestas estereotipadas (Navarro et al. 2016). Además, el SSAQ incluye tres ítems socio-demográficos, dada su relevancia en la investigación de las actitudes hacia la ciencia (Potvin y Hasni 2014): edad, curso y sexo.

El SSAQ utiliza una escala Likert de 1 a 5 que permite al alumnado mostrar su grado de acuerdo con los ítems mostrados en el anexo. Así, se establece el rango: muy desacuerdo, desacuerdo, parcialmente de acuerdo, de acuerdo y muy de acuerdo.

\section{¿Medir la actitud hacia la ciencia? Análisis comparativo del SSAQ, TOSRA y S-SSAS}

Los hallazgos evidenciados por Blalock, Lichtenstein, Owen, Pruski, Marshall y Toepperwein (2008) y Potvin y Hasni (2014) en sus trabajos de revisión, apuntan a la elevada cantidad de instrumentos desarrollados para medir la AC que no presentan datos relativos a su validez y fiabilidad. En este sentido, Tytler (2014) desaconseja utilizar instrumentos para medir la AC del alumnado sin disponer de su validez de constructo previamente, pues sería contraproducente para esta línea de investigación, dada la importancia de las propiedades psicométricas de los instrumentos que miden actitudes. Por tanto, la confrontación del SSAQ frente a los instrumentos TOSRA (Navarro et al. 2016) y S-SSAS (Toma y Meneses-Villagrá 2019) obedece a dos cuestiones principales: (1) están traducidos y validados en español; y (2) han aportado una validación de constructo sólida.

Desde la perspectiva conceptual, tanto Navarro et al. (2016) como Toma y Meneses-Villagrá (2019) asumen y describen la complejidad del constructo AC, además de reseñar la importancia de construir instrumentos multidimensionales para medir dicho constructo (Tytler 2014). Asimismo, ambos utilizan los distintos ámbitos de las actitudes del alumnado relacionadas con la ciencia sugeridos por Klopfer (1971): (1) ciencia y científicos; (2) actitud hacia la indagación; (3) adopción de actitudes científicas; (4) disfrute con el aprendizaje de la ciencia; (5) interés por el aprendizaje de la ciencia; y (6) interés en estudiar carreras científicas. Esta clasificación es utilizada por los autores para posicionar al instrumento en cuestión, así que Navarro et al. (2016) sitúan al TOSRA como un instrumento para medir la AC en general del alumnado, mientras Toma y Meneses-Villagrá (2019) optan por la actitud hacia la ciencia escolar.

Desde la perspectiva estructural, hemos de atender a las dimensiones que conforman a los instrumentos objeto de comparación, así como a su soporte teórico. La versión española del TOSRA validada por Navarro et al. (2016), al igual que la versión original de Fraser (1978), incorpora las dimensiones: (1) implicaciones sociales de la ciencia; (2) normalidad de los científicos (3) actitud hacia la investigación científica; (4) adopción de actitudes científicas; (5) 
disfrute en las clases de ciencias; (6) interés en la ciencia; y (7) interés profesional en la ciencia. El soporte teórico de las dimensiones del TOSRA responde a la clasificación establecida por Klopfer (1971) para describir los ámbitos de la AC. Contrariamente, la versión española del SSAS (S-SSAS) de Toma y Meneses-Villagrá (2019) contempla las dimensiones: (1) intención de matricularse en ciencias; (2) disfrute con la ciencia escolar; (3) dificultad percibida de la ciencia escolar; (4) percepción de la autoeficacia en la ciencia escolar; (5) utilidad de la ciencia para las carreras; y (6) relevancia de la ciencia escolar. Estos autores, junto con los autores de la versión original (Kennedy, Quinn y Taylor 2016), fundamentan las dimensiones descritas a partir de aquellos indicadores establecidos por distintos estudios para medir la AC del alumnado: disfrute, autoeficacia, dificultad percibida, relevancia y utilidad de la ciencia (Osborne et al. 2003; Potvin y Hasni 2014) y otras creencias conductuales relacionadas con su involucración en el aprendizaje de las ciencias (Blalock et al. 2008; Said et al. 2016; Summers y Abd-El-Khalick 2018).

Tras el análisis conceptual y estructural de ambos instrumentos, hemos de concluir que: (1) partiendo de la clasificación de Klopfer (1971), el soporte teórico proporcionado en las secciones anteriores y a la luz de las características del SSAQ, este estaría alineado con la medición de la actitud hacia la ciencia escolar (considerado por Osborne et al. 2003 el mejor predictor de la AC en general); y (2) la adaptación del SSAQ al constructo de AC, así como la idoneidad de la misma, queda justificada dada la semejanza estructural entre el SSAQ y los cuestionarios analizados, especialmente el S-SSAS. En este sentido, los indicadores propuestos por Speering y Rennie (1996), que fundamentan a este instrumento, están en consonancia con los utilizados por Toma y Meneses-Villagrá (2019). Por tanto, desde una perspectiva teórica, parece plausible utilizar los indicadores propuestos por Osborne et al. (2003), Potvin y Hasni (2014), Blalock et al. (2008), Said et al. (2016), Summers y Abd-El-Khalick (2018) y Speering y Rennie (1996) para medir la AC del alumnado dada su naturaleza evaluativa, fundamentada en las creencias y percepciones individuales del alumnado, y dada la relación directa entre creencia, afecto y actitud (Fishbein y Ajzen 1975).

Si avanzamos en la comparativa iniciada, podemos observar que los instrumentos TOSRA y SSSAS, diseñados originalmente por Fraser (1978) y Kennedy et al. (2016), poseen características muy dispares, acordes a la etapa educativa a la que van dirigidas. Así, el TOSRA está dirigido a la etapa de Educación Secundaria, mientras que el SSAS se dirige a alumnado de Educación Primaria. La población diana de estos instrumentos determina de manera muy marcada sus características, de forma que el TOSRA incorpora 70 ítems en una escala Likert de 1 a 5, mientras que el SSAS utiliza 10 ítems en los que se mezcla la escala Likert de 1 a 5 para mostrar el grado de acuerdo con escalas de diferencial semántico. Tan es así que la longitud del primero como la sencillez del segundo son ejemplos de características que los hacen instrumentos con una población estudiantil muy delimitada. Por tanto, el SSAQ, al utilizar un número de ítems intermedio $\left(\mathrm{n}^{\circ}\right.$ ítems $\left.=20\right)$ con una escala Likert de 1 a 5 al igual que el TOSRA y el SSAS, podría aportar la operatividad necesaria para medir la AC del alumnado de los últimos cursos de Educación Primaria (10-12 años) y ESO (12-16 años). Así, la población de estudiantes a la que podría dirigirse el SSAQ lo sitúan como un instrumento operativo e idóneo para medir (longitudinal o transversalmente) la actitud del alumnado hacia la ciencia durante el tránsito de la Educación Primaria a la Secundaria, momento donde más se acusa el deterioro de la AC (Pell y Jarvis 2001; Vázquez y Manassero 2008), ya que existe consenso en la comunidad científica al afirmar que las actitudes positivas del alumnado hacia la ciencia descienden según este avanza en el sistema educativo (Osborne et al. 2003; VedderWeis y Fortus 2011). No obstante, hemos de ser cautos y tener en cuenta que esta validación se ha realizado en alumnado de Educación Secundaria. 


\section{Procedimiento}

Una vez la investigación fue aprobada por la Administración educativa, el instituto y los tutores legales del alumnado, se contactó con los profesores de las asignaturas de ciencias (entiéndanse: Biología, Geología, Física y Química) y con los tutores de aquellos cursos en los que no se imparten dichas asignaturas, como ocurre con cuarto curso de ESO en las modalidades de Ciencias Sociales, Humanidades y Artes. Los datos fueron recogidos por el primer autor entre diciembre de 2017 y enero de 2018, quien concertó cita previa con los ocho docentes contactados.

Los cuestionarios se administraron a los estudiantes en su clase habitual y en ausencia del docente. El procedimiento utilizado fue idéntico en las once clases a las que se accedió para recabar los datos, de manera que el investigador explicó en qué consistía el cuestionario y el modo en el que debía cumplimentarse. Tras ello, se realizaba el reparto de los cuestionarios para que el alumnado pudiera leerlo detenidamente y consultar aquellas dudas que le surgiesen respecto a la comprensión de los ítems. En este sentido, cabe señalar que dos alumnos (inferior al 1\% de la muestra objeto de estudio) manifestaron dudas en relación al ítem 6 por no conocer el significado de «incrementarse». Tras la resolución de aquellas, el alumnado procedió a la cumplimentación del cuestionario, tarea que ocupó entre 12 y 15 minutos. Finalmente, los participantes levantaban la mano para indicar que habían finalizado (tal y como se les indicó al inicio), permitiendo al investigador recoger los cuestionarios uno a uno y revisar la cumplimentación correcta de los mismos (en aquellos casos en los que el alumno olvidó responder a algún ítem, se le solicitó releerlo para responder).

\section{Análisis de los datos}

El análisis estadístico se realizó con IBM SPSS Statistics 20.0 y AMOS 24.0. En relación a la estadística descriptiva, se calculó la media y la desviación típica de cada uno de los ítems. También, se analizó la multicolineidad de los ítems a partir del estadístico rho de Spearman. Además, antes de iniciar los análisis factoriales, se valoró la normalidad univariada y multivariada de los datos a partir de los estadísticos asimetría y curtosis para el primer caso, y del coeficiente de Mardia para el segundo. En cuanto a la estadística inferencial, se utilizó la prueba U de Mann-Whitney para analizar las diferencias según el sexo del alumnado.

En cuanto a los resultados obtenidos en los estadísticos asimetría y curtosis, los ítems 1 y 5 excedieron levemente el rango $-1 /+1$ en dichos estadísticos y en el coeficiente de Mardia (52.62, p. < .001), lo que parece indicar que no se cumplen los supuestos de normalidad univariada en todos los ítems (Ferrando y Anguiano-Carrasco 2010) y multivariada (Ullman 2006). Por tanto, en la validación de constructo a través del AFE y AFC se empleó el método Mínimos Cuadrados no Ponderados (ULS de sus siglas en inglés), el cual es el más recomendado para analizar ítems de naturaleza ordinal que no obedecen a una distribución normal (Forero, Maydeu-Olivares y Gallardo-Pujol 2009), además de proporcionar soluciones factoriales estables incluso en tamaños muestrales pequeños (Jung 2013).

Finalmente, el AFC se ejecutó según el procedimiento sugerido por Kline (2005): (1) especificación del modelo; (2) identificación; (3) estimación de parámetros; y (4) evaluación del ajuste. La evaluación del ajuste del modelo estructural propuesto en el SSAQ se realizó a partir de los índices de ajuste proporcionados conforme al método ULS: (1) $\chi^{2} /$ g.l. (grados de libertad); (2) el índice gamma (GFI); (3) el residuo cuadrático medio estandarizado (RMR); y (4) el índice normado de ajuste (NFI). Además, la fiabilidad se evalúo a partir del alfa de Cronbach $(\alpha)$ y la fiabilidad compuesta (FC) junto con la varianza media extraída (VME), calculados para cada dimensión. La interpretación de todos estos parámetros se realizó conforme a las pautas mostradas en la Tabla 2. 
Tabla 2. Interpretación de parámetros.

\begin{tabular}{|c|c|c|c|}
\hline Autores & Parámetro & Valores excelentes & Valores aceptables \\
\hline Schermelleh-Engel, & $\chi^{2} /$ g.l. & $0 \leq \chi^{2} /$ g.l. $\leq 2$ & $2 \leq \chi^{2} /$ g.l. $\leq 3$ \\
\hline \multirow[t]{3}{*}{ Moosbrugger y Müller (2003) } & RMR & $0 \leq \mathrm{RMR} \leq .05$ & $.05<\mathrm{RMR} \leq .10$ \\
\hline & NFI & $.95 \leq \mathrm{NFI} \leq 1.00$ & $.90 \leq \mathrm{NFI}<.95$ \\
\hline & GFI & $.95 \leq \mathrm{GFI} \leq 1.00$ & $.90 \leq$ GFI $<.95$ \\
\hline DeVellis (2003) & $\alpha$ & $.80<\alpha \leq 1.00$ & $.65 \leq \alpha \leq .80$ \\
\hline Hair, Black, Babib y & $\mathrm{FC}$ & - & $\mathrm{FC} \geq .70$ \\
\hline Anderson (2010) & VME & - & $\mathrm{VME} \geq .50$ \\
\hline
\end{tabular}

\section{Resultados}

Los resultados obtenidos en la Tabla 3 parecen indicar, en líneas generales, que la AC del alumnado participante es aceptable. Además, según los resultados arrojados por la prueba no paramétrica U de Mann-Whitney no se detectaron diferencias significativas entre alumnos y alumnas en ninguno de los ítems del SSAQ.

Tabla 3. Medias (M) y desviaciones típicas (DT) de los ítems, según sexo del alumnado y valor p. de la prueba U de Mann-Whitney.

\begin{tabular}{|c|c|c|c|c|c|c|c|}
\hline \multirow[b]{2}{*}{ Ítem } & \multicolumn{2}{|c|}{ General } & \multicolumn{2}{|c|}{ Alumnos } & \multicolumn{2}{|c|}{ Alumnas } & \multirow[b]{2}{*}{ p. } \\
\hline & $\mathbf{M}$ & DT & $\mathbf{M}$ & DT & $\mathbf{M}$ & DT & \\
\hline 1. Pienso que la ciencia es un tema interesante & 4.04 & 0.813 & 4.05 & 0.819 & 4.03 & 0.809 & .885 \\
\hline $\begin{array}{l}\text { 2. En clase de ciencias obtengo respuestas a preguntas } \\
\text { que me intrigan }\end{array}$ & 3.64 & 0.906 & 3.68 & 0.881 & 3.59 & 0.932 & .614 \\
\hline $\begin{array}{l}\text { 3. En clase de ciencias puedo expresar mis propias } \\
\text { ideas }\end{array}$ & 3.47 & 0.977 & 3.48 & 0.942 & 3.46 & 1.016 & .893 \\
\hline $\begin{array}{l}\text { 4. Puedo tener éxito en la ciencia sin la ayuda del } \\
\text { profesor }\end{array}$ & 2.37 & 0.953 & 2.41 & 0.986 & 2.33 & 0.921 & .530 \\
\hline $\begin{array}{l}\text { 5. Las asignaturas de ciencias no deberían ser } \\
\text { obligatorias en la escuela }\end{array}$ & 1.98 & 1.114 & 1.96 & 1.151 & 2.01 & 1.079 & .441 \\
\hline $\begin{array}{l}\text { 6. El número de horas por semana de ciencias debería } \\
\text { incrementarse }\end{array}$ & 2.71 & 1.101 & 2.66 & 1.061 & 2.77 & 1.142 & .519 \\
\hline 7. Las clases de ciencias me fascinan & 3.43 & 1.099 & 3.43 & 1.113 & 3.43 & 1.089 & .962 \\
\hline 8. Las clases de ciencias me aburren & 2.16 & 1.177 & 2.13 & 1.190 & 2.19 & 1.166 & .588 \\
\hline $\begin{array}{l}\text { 9. Es importante para mí entender lo que se enseña en } \\
\text { clase de ciencias }\end{array}$ & 3.99 & 0.942 & 3.89 & 0.950 & 4.09 & 0.927 & .085 \\
\hline 10. Las lecciones de ciencias son fáciles de estudiar & 2.81 & 1.055 & 2.78 & 1.029 & 2.85 & 1.083 & .527 \\
\hline 11. Me divierto aprendiendo ciencias & 3.39 & 1.081 & 3.48 & 1.021 & 3.29 & 1.135 & .196 \\
\hline 12. En el futuro me gustaría ser científico & 2.40 & 1.248 & 2.45 & 1.282 & 2.35 & 1.216 & .593 \\
\hline $\begin{array}{l}\text { 13. Los estudios científicos me permiten entender } \\
\text { fenómenos cotidianos }\end{array}$ & 3.80 & 0.934 & 3.79 & 0.946 & 3.81 & 0.925 & .823 \\
\hline $\begin{array}{l}\text { 14. Confío en mis habilidades para tener éxito en el } \\
\text { estudio de las ciencias }\end{array}$ & 3.40 & 1.096 & 3.42 & 1.048 & 3.37 & 1.146 & .799 \\
\hline 15. Ayudo a los demás en las clases de ciencias & 3.03 & 1.050 & 3.04 & 1.022 & 3.03 & 1.082 & .963 \\
\hline $\begin{array}{l}\text { 16. Me gusta leer artículos y ver programas que tratan } \\
\text { temas científicos }\end{array}$ & 2.39 & 1.245 & 2.51 & 1.301 & 2.28 & 1.179 & .180 \\
\hline $\begin{array}{l}\text { 17. Me interesan las explicaciones de los fenómenos } \\
\text { científicos }\end{array}$ & 3.51 & 1.108 & 3.55 & 1.142 & 3.47 & 1.076 & .415 \\
\hline $\begin{array}{l}\text { 18. Creo que la comprensión de la ciencia es } \\
\text { importante para todos }\end{array}$ & 2.72 & 0.940 & 3.84 & 0.947 & 3.91 & 0.936 & .548 \\
\hline 19. Es difícil para mí aprender ciencia & 2.72 & 1.161 & 2.80 & 1.164 & 2.64 & 1.158 & .347 \\
\hline 20. La ciencia no tiene conexión con mi vida & 2.02 & 1.067 & 2.02 & 1.110 & 2.01 & 1.027 & .888 \\
\hline
\end{tabular}

El análisis pormenorizado, ítem a ítem, nos permite afirmar que el alumnado considera importante la ciencia escolar y disfruta con su aprendizaje. Además, parece existir cierta cohesión entre los resultados. A modo de ejemplo podemos observar los resultados de los ítems 5 y 18, estos muestran cómo el alumnado es consciente de la importancia de una formación básica en ciencias para toda la ciudadanía y que ello parte de cursar asignaturas de 
ciencias en los niveles educativos obligatorios. A pesar de esto, la dispersión de los resultados es amplia, dado que todos los ítems evidencian una desviación típica próxima o superior a uno. No obstante, de los resultados mostrados en la Tabla 3, aquellos obtenidos en los ítems 4, 12 y 16 podrían ser los más relevantes. Así, parece generalizado entre el alumnado que fuera del contexto escolar (item 16) o al término de los niveles educativos obligatorios (ítem 12) su relación e interés por la ciencia podría descender.

Por otro lado, la correlación bivariada realizada sobre los 20 ítems que conforman el SSAQ arroja unos valores de rho de Spearman significativos $(p .<.05$ ) en el $98 \%$ de las 190 combinaciones posibles e inferiores a .85 en todas ellas, de forma que no existe multicolineidad entre los ítems, es decir, los ítems del SSAQ no son redundantes según las aportaciones de Kline (2005).

\section{Validación de constructo (AFE)}

La ejecución del AFE arrojó una solución factorial compuesta por cuatro factores, correspondiéndose con las cuatro dimensiones establecidas en el SSAQ y explicando el 46.65\% de la varianza (D1: 36.60\%; D2: 4.73\%; D3: 3.13\%; D4: 2.19\%). Estos fueron retenidos según el criterio de Kaiser (autovalores superiores a 1) y conforme a la pendiente del gráfico de sedimentación (Cattell, 1966) (Figura 1).

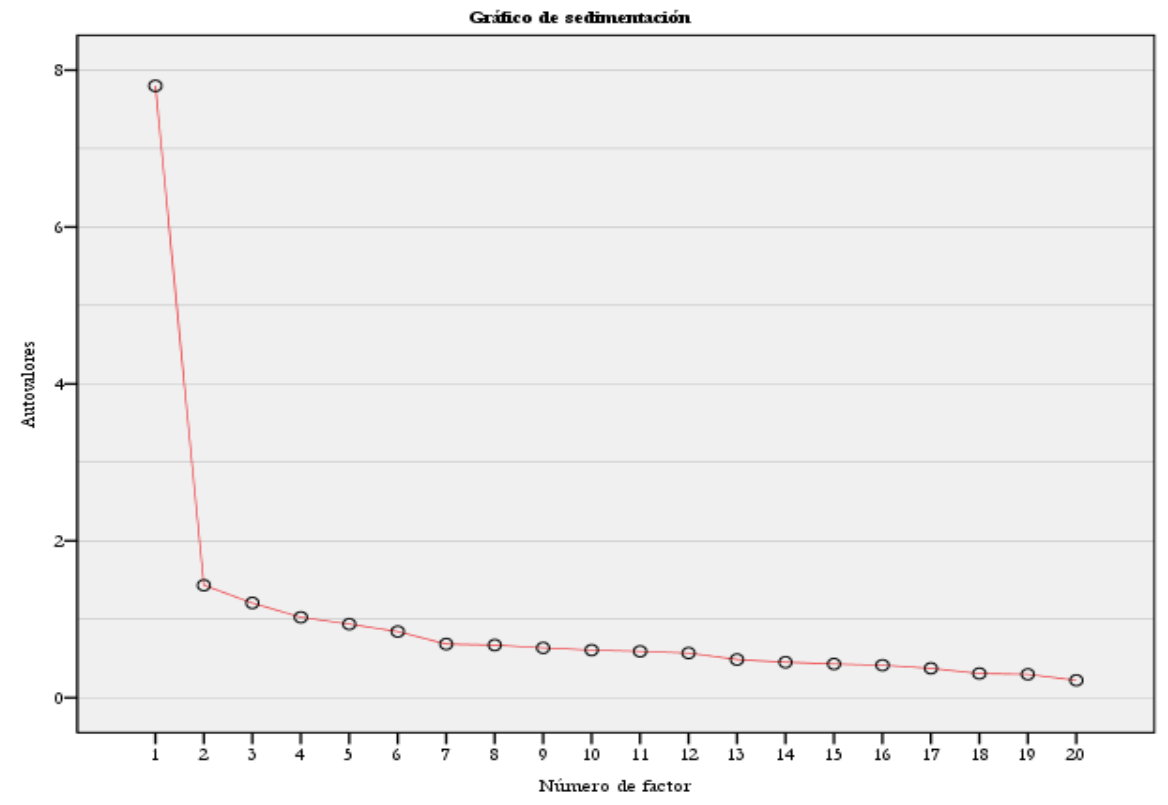

Figura 1. Gráfico de sedimentación con los cuatro factores retenidos en el SSAQ.

El test de adecuación muestral de Kaiser-Meyer-Olkin $(\mathrm{KMO}=0.93)$ y la prueba de esfericidad de Barlett (p. < .001) mostraron resultados satisfactorios. Ello, unido con las comunalidades obtenidas, comprendidas entre los valores 0.29 y 0.71 , la distribución de cinco ítems por dimensión y una muestra superior a 200 alumnos, parecen generar una solución factorial exploratoria aceptable (Lloret-Segura et al. 2014). Además, las cargas factoriales de los ítems respecto a la dimensión designada (Tabla 4) fueron superiores al mínimo de .30 establecido por Bandalos y Finney (2010). 
Tabla 4. Cargas factoriales obtenidas en el AFE con rotación Oblimin directo.

\begin{tabular}{|c|c|c|c|c|c|}
\hline Ítem & & D1 & D2 & D3 & D4 \\
\hline 1 & Pienso que la ciencia es un tema interesante & .331 & .202 & .482 & .286 \\
\hline 2 & En clase de ciencias obtengo respuestas a preguntas que me intrigan & .373 & .043 & .314 & .411 \\
\hline 3 & En clase de ciencias puedo expresar mis propias ideas & .299 & .090 & .289 & .302 \\
\hline 4 & Puedo tener éxito en la ciencia sin la ayuda del profesor & .255 & .370 & .157 & .242 \\
\hline 5 & Las asignaturas de ciencias no deberían ser obligatorias en la escuela & .578 & .124 & .340 & .423 \\
\hline 6 & El número de horas por semana de ciencias debería incrementarse & .631 & .288 & .336 & .345 \\
\hline 7 & Las clases de ciencias me fascinan & .375 & .370 & .472 & .223 \\
\hline 8 & Las clases de ciencias me aburren & .297 & .272 & .318 & .204 \\
\hline 9 & Es importante para mí entender lo que se enseña en clase de ciencias & .609 & .020 & .460 & .451 \\
\hline 10 & Las lecciones de ciencias son fáciles de estudiar & .461 & .687 & .382 & .230 \\
\hline 11 & Me divierto aprendiendo ciencias & .450 & .421 & .535 & .282 \\
\hline 12 & En el futuro me gustaría ser científico & .382 & .324 & .294 & .471 \\
\hline 13 & Los estudios científicos me permiten entender fenómenos cotidianos & .441 & .160 & .213 & .359 \\
\hline 14 & Confío en mis habilidades para tener éxito en el estudio de las ciencias & .330 & .404 & .267 & .248 \\
\hline 15 & Ayudo a los demás en las clases de ciencias & .304 & .350 & .276 & .177 \\
\hline 16 & Me gusta leer artículos y ver programas que tratan temas científicos & .557 & .206 & .229 & .563 \\
\hline 17 & Me interesan las explicaciones de los fenómenos científicos & .374 & .062 & .419 & .369 \\
\hline 18 & Creo que la comprensión de la ciencia es importante para todos & .540 & .028 & .340 & .393 \\
\hline 19 & Es difícil para mí aprender ciencia & .391 & .683 & .272 & .307 \\
\hline 20 & La ciencia no tiene conexión con mi vida & .494 & .263 & .225 & .587 \\
\hline
\end{tabular}

\section{Validación de constructo (AFC)}

El ajuste del modelo teórico del SSAQ, compuesto por cuatro variables latentes (dimensiones) medidas por cinco variables observables (items) cada una, se muestra en la Tabla 5.

Tabla 5. Índices de ajuste obtenidos en el modelo de medida.

\begin{tabular}{lccccc}
\hline \multicolumn{1}{c}{ Modelo } & $\chi^{2} /$ g.l. & RMR & GFI & NFI \\
\cline { 1 - 2 } $\begin{array}{l}\text { Scholar Science Attitude } \\
\text { Questionnaire }\end{array}$ & 1.028 & .056 & .986 & .980 \\
\hline
\end{tabular}

Los resultados obtenidos indican un ajuste adecuado del modelo teórico propuesto en el SSAQ, de modo que el resultado de 1.028 obtenido en $\chi^{2} /$ g.l., .986 en el caso del GFI o .980 para NFI se consideran excelentes según Schermelleh-Engel et al. (2003), mientras que el valor de RMR (.056) es aceptable según el criterio propuesto por los autores anteriores. Además, los ítems utilizados para medir las diferentes variables latentes presentan cargas factoriales adecuadas para cada una de ellas, siendo todas iguales o superiores a .30 (Bandalos y Finney 2010). Ello se puede observar en la Figura 2, que leída de izquierda a derecha ilustra el modelo teórico propuesto para el SSAQ, mostrándose las covarianzas entre las dimensiones, así como las cargas factoriales y los errores de medida de cada ítem.

Finalmente, los valores de alfa de Cronbach, FC y VME calculados para cada una de las dimensiones (Tabla 6) arrojan unos valores aceptables en los dos primeros casos, al oscilar entre los valores .66 y .88 en el caso del alfa de Cronbach (DeVellis 2003) y ser ampliamente superiores a .70 en el caso de la FC. No obstante, los valores obtenidos para la VME, salvo en el caso de la D3 (.62), no superan el valor .50 recomendado (Hair et al. 2010). Además, el alfa de Crobanch obtenido en la totalidad de los ítems corrobora la robustez de la fiabilidad del SSAQ, al obtenerse un valor de .91.

Tabla 6. Valoración de la consistencia interna del SSAQ.

\begin{tabular}{|c|c|c|c|c|c|}
\hline Dimensión & Nombre & $\mathrm{N}^{\mathrm{o}}$ de Ítems & $\alpha$ & FC & $\overline{\text { VME }}$ \\
\hline D1 & $\begin{array}{l}\text { Importancia de la ciencia para } \\
\text { el estudiante }\end{array}$ & 5 & .74 & .83 & .37 \\
\hline $\mathrm{D} 2$ & Autoeficacia & 5 & .70 & .82 & .33 \\
\hline D3 & Interés y disfrute & 5 & .88 & .83 & .62 \\
\hline D4 & $\begin{array}{l}\text { Conexión de la ciencia con la } \\
\text { vida diaria del estudiante }\end{array}$ & 5 & .66 & .83 & . \\
\hline
\end{tabular}




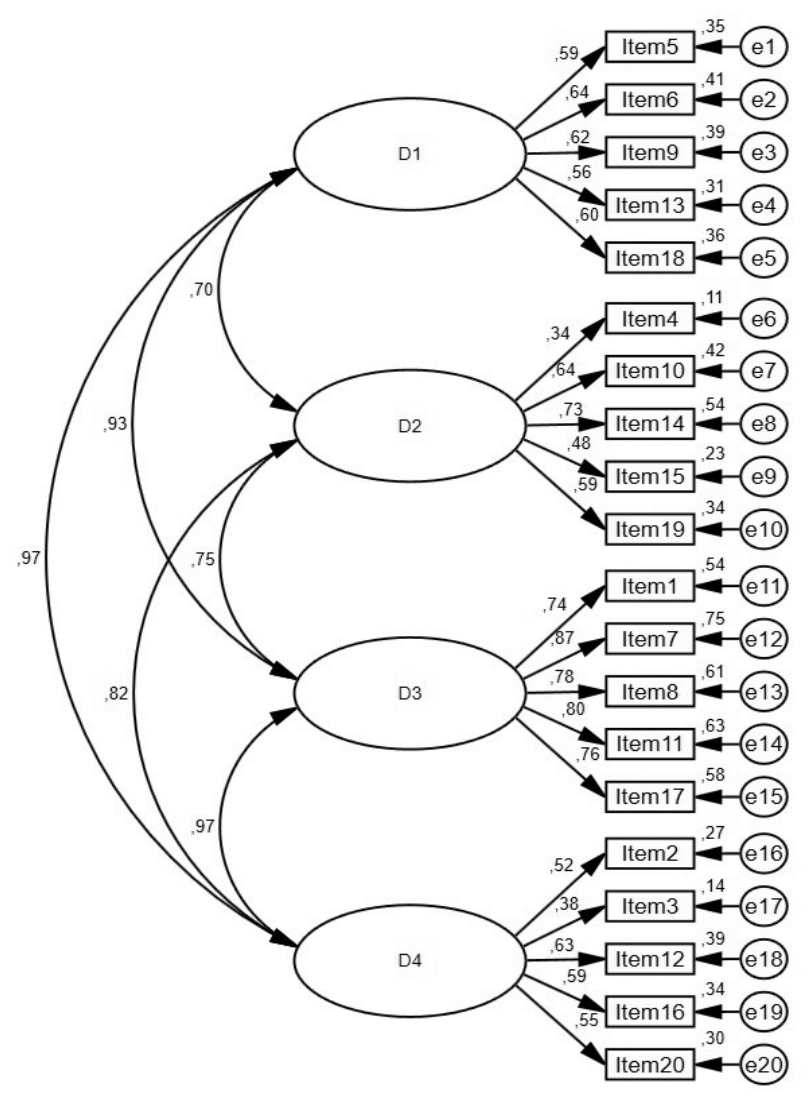

Figura 2. Análisis estructural del SSAQ (N = 259).

\section{Discusión}

Tal como adelantamos, el presente trabajo se había propuesto dos objetivos. El primero planteaba traducir al español, adaptar y proveer de soporte teórico el cuestionario de Barak et al. (2011), el cual pasaría a llamarse SSAQ al término de dicho proceso. El segundo fue determinar si la estructura del instrumento era correcta a través de la implementación de un AFE y un AFC, este último ejecutado a partir de un modelo de medida. Transversalmente, se analizó la puntuación media global y según el sexo del alumnado de cada ítem. Esto nos lleva a discutir los datos desde dos perspectivas: aquella que atiende a los datos descriptivos e inferenciales presentados en la Tabla 3 referentes a la AC del alumnado y aquella centrada en las propiedades psicométricas del SSAQ.

En cuanto a la AC del alumnado, se obtuvieron en líneas generales unos resultados satisfactorios, pues el alumnado manifestó un interés y disfrute hacia el aprendizaje de las ciencias aceptable, igualmente parecen reconocer la importancia de la ciencia escolar en el ámbito personal y social. No obstante, la intención de comportamiento manifestada por el alumnado, vinculada a la conexión de la ciencia con la vida diaria de este, no se corresponde con la importancia que atribuyen a la ciencia. En este sentido, Koballa (1988a) ya apuntaba a este recurrente conflicto cognitivo que presenta el alumnado, pues otros autores como Jenkins y Nelson (2005) también reflejaron en su estudio que generalmente el alumnado es capaz de reconocer la importancia de las ciencias pero que, igualmente, decide no continuar con estudios superiores de carácter científico. Por otro lado, a pesar de que el alumnado manifiesta confianza en sus capacidades para afrontar el aprendizaje de las ciencias, el grado de autoeficacia de este parece estar ligado a la labor docente (ver ítem 4 en Tabla 3). Hecho que 
se corresponde con los hallazgos de Usher y Pajares (2009), quienes identifican un fuerte vínculo entre la experiencia escolar (incluida en esta la actuación didáctica del docente) del alumnado y la percepción de sus habilidades para completar satisfactoriamente un objetivo académico.

En relación a la aparente moderación del sexo en la actitud del alumnado hacia la ciencia, en este trabajo no se identificaron diferencias significativas en ninguno de los ítems del SSAQ en cuanto al sexo del alumnado. Hecho que refleja la discusión generada en esta línea de investigación respecto a esta variable, de forma que los resultados obtenidos son contrarios a aquellos evidenciados por Pro y Pérez-Manzano (2014), Denessen et al. (2015) o De Witt y Archer (2015), pero similares a los presentados por Akpinar et al. (2009), Navarro et al. (2016), Toma y Greca (2018) o Toma y Meneses-Villagrá (2019).

Respecto a la validez de constructo del SSAQ, se podría determinar que se ajusta de manera aceptable al modelo teórico propuesto y, por tanto, podría ser una herramienta válida y fiable para medir la AC del alumnado hispanohablante de Educación Secundaria. Ello puede sostenerse a partir de los resultados mostrados en el AFE, en el que se obtuvo un $46.65 \%$ de la varianza explicada, resultado ligeramente superior al obtenido por Navarro et al. (2016) en la validación de la versión española del TOSRA (45\%). Además, el AFC ofrece unos valores adecuados en los índices de ajuste $\chi^{2} /$ g.l. (1.028), GFI (.986), RMR (.056) y NFI (.980) (Schermelleh-Engel et al. 2003) obtenidos a partir del método ULS, los cuales parecen mejorar ostensiblemente el ajuste del modelo teórico del TOSRA en la población hispanohablante $\left(\chi^{2} /\right.$ g.l. $\left.=3.57 ; \mathrm{GFI}=.644 ; \mathrm{RMR}=.185\right)($ Navarro et al. 2016).

Finalmente, en cuanto a la fiabilidad del SSAQ, los resultados obtenidos en $\alpha$ y FC auguran estabilidad en la medición de los constructos contemplados, pues ambos estadísticos presentan valores aceptables en todas las dimensiones y a nivel global. En comparación con los instrumentos S-SSAS y la versión española del TOSRA, y conociendo que el alfa de Cronbach se ve afectado por el número de ítems del instrumento (a mayor cantidad de ítems mayor $\alpha$ ), el SSAQ obtuvo un resultado global de .91 ligeramente inferior al obtenido por el TOSRA (.94) (Navarro et al. 2016) y superior al presentado por el S-SSAS (.70) (Toma y Meneses-Villagrá, 2019). Sin embargo, los valores de VME calculados no nos permiten certificar la validez convergente de todos las dimensiones del SSAQ, dado que solo la D3 (.62) presenta un valor aceptable según Hair et al. (2010), mientras que las dimensiones 1 (.37), 2 (.33) y 4 (.30) no superan al valor mínimo recomendado (.50). Por el contrario, de acuerdo a las directrices y recomendaciones de Brown (2006), los coeficientes de correlación rho de Spearman (dada la no normalidad de los datos) de las dimensiones que componen al SSAQ arrojan valores inferiores al límite establecido de .80 (Tabla 7), por lo que se confirma la validez discriminante de aquellas.

Tabla 7. Correlación bivariada rho de Spearman de las dimensiones del SSAQ.

\begin{tabular}{lcccc}
\hline & D1 & D2 & D3 & D4 \\
\hline D1. Importancia de la ciencia para el estudiante & 1 & $.191^{* *}$ & $.560^{* *}$ & $.459^{* *}$ \\
D2. Autoeficacia & & 1 & $.262^{* *}$ & $.287^{* *}$ \\
D3. Interés y disfrute & & & 1 & $.591^{* *}$ \\
D4. Conexión de la ciencia con la vida diaria del estudiante & & & 1 \\
\hline$* *$ Valor p. $<.01$. & & & &
\end{tabular}

\section{Limitaciones}

Las limitaciones del estudio responden principalmente a la muestra de alumnos seleccionada, dado que se realizó de forma intencional y eludiendo el muestreo probabilístico. Hecho que podría presentar dos problemas: la no representatividad y la atenuación debida a la restricción de rango (Ferrando y Anguiano-Carrasco 2010). Además, el SSAQ presenta una limitación en 
su validez de constructo, debido a que no se contempló la validez concurrente (comparación con otros instrumentos ya validados), hecho que sí realizaron Toma y Meneses-Villagrá (2019) y Navarro et al. (2016) en los instrumentos S-SSAS y TOSRA, respectivamente.

Por otra parte, a pesar de apoyarse en la «Teoría de la Acción Razonada» (Fishbein y Azjer 1975), el SSAQ no contempla la influencia del entorno (familia, amigos...) que incide directamente en la génesis de creencias, denominadas como «creencias referentes a normas sociales subjetivas» en la teoría descrita. Por tanto, parece que obviar estas creencias en el SSAQ podría parcializar la medición de la AC. Sin embargo, se ha podido mostrar estadísticamente que la formación de actitudes hacia la ciencia se debe mayoritariamente a la percepción del estudiante sobre la relevancia y utilidad de la misma, prevaleciendo sobre las presiones sociales que le rodean (Koballa 1988b). En este sentido, pero desde una perspectiva cualitativa, Rodríguez-Menéndez, Peña-Calvo e Inda-Caro (2016) identificaron en una muestra de estudiantes del $4^{\circ}$ curso de ESO y $1^{\circ}$ de Bachillerato la denominada «ideología del gusto innato», de modo que estos estudiantes evidencian que su gusto e interés por continuar estudiando ciencia parte de ellos mismos y es ajeno a cualquier influencia social.

\section{Implicaciones educativas}

El cuestionario SSAQ, junto con los analizados S-SSAS y TOSRA, se presentan como instrumentos válidos y fiables para medir la AC del alumnado, bien de Educación Primaria (caso del S-SSAS) o bien de Educación Secundaria (SSAQ y TOSRA). No obstante, vistas las cualidades y características del SSAQ, podría ser utilizado en los últimos cursos de la etapa de Educación Primaria, aunque para garantizar su utilidad y viabilidad en dicha etapa educativa se necesita ampliar la validación que aquí se presenta.

Por otra parte, el SSAQ es un instrumento de lápiz y papel que puede ser utilizado de forma rápida y operativa en el aula, al no requerir su cumplimentación más de 15 minutos (con alumnado de Educación Secundaria). Además, es importante tener en cuenta que las actitudes hacia la ciencia están consideradas por el MECD (2014a; 2014b) como una variable a considerar y a desarrollar durante el proceso de enseñanza-aprendizaje. Por tanto, el SSAQ se postula como una herramienta válida y fiable para medir la $\mathrm{AC}$ del alumnado en el contexto español e iberoamericano.

\section{Conclusiones}

El desarrollo y la validación estructural del SSAQ nos permite alcanzar una serie de conclusiones finales en relación con los objetivos iniciales:

Primera, la traducción inglés-español del instrumento fue realizada por personal capacitado para ello, desarrollando un proceso de traducción bidireccional por dos docentes bilingües diferentes, con lo cual se ha garantizado la fiabilidad de la traducción. Además, el proceso de adaptación se ha fundamentado teóricamente y ha incluido descripciones claras de las dimensiones que componen el SSAQ, en aras de dotar de un soporte teórico a dicho instrumento.

Segunda, la estructura del SSAQ resulta válida y fiable para medir la actitud hacia la ciencia del alumnado de ESO, dado que no solamente se ajusta al modelo teórico establecido, sino que también evidencia una elevada correspondencia con el constructo de actitud. Además, estas características, unidas a lo reducido del número de ítems frente a otros cuestionarios de amplia difusión, permite a nuestro juicio una aplicación más operativa en las aulas, dado que atenúa el efecto fatiga en este tipo de instrumentos. 
Finalmente, este trabajo podría considerarse como el inicio del proceso de validación del SSAQ, pues en trabajos futuros sería pertinente replicar una validación estructural en alumnos de $5^{\circ}-6^{\circ}$ de Educación Primaria y Educación Secundaria de forma conjunta, a fin de ampliar la muestra de estudio y certificar su idoneidad para la etapa de Primaria. Además, sería apropiado analizar la validez concurrente del cuestionario, así como validarlo en su versión inglesa, de forma que para tales fines se adjuntan en el Anexo los ítems que componen el SSAQ tanto en español como en inglés.

\section{Agradecimientos}

A la Universidad de Granada, la Junta de Andalucía y al Fondo Social Europeo por la financiación del contrato ${ }^{\circ}$ 6161 destinado a la contratación de joven personal investigador y al grupo de investigación HUM-613, por participar en su formación.

\section{Referencias}

Aguilera D., Perales F.J. (2018a) What Effects Do Didactic Interventions Have on Students' Attitudes Towards Science? A Meta-Analysis. Research in Science Education, 1-25. DOI: $10.1007 /$ s11165-018-9702-2

Aguilera D., Perales F.J. (2018b) El libro de texto, las ilustraciones y la actitud hacia la Ciencia del alumnado: percepciones, experiencias y opiniones del profesorado. Enseñanza de las ciencias 36(3), 41-58.

Ajzen I., Fishbein M. (2005) The influence of attitudes on behavior. In D. Albarracín, B.T. Johnson, M.P. Zanna (Eds.), The handbook of attitudes (pp. 173-221). Mahwah, NJ: Erlbaum.

Akpinar E., Yildiz E., Tatar N., Ergin O. (2009) Students' attitudes toward science and technology: an investigation of gender, grade level, and academic achievement. Procedia Social and Behavioral Sciences 1(1), 2804-2808.

Ali M.M., Yager R.E., Hacieminoglu E., Caliskan I. (2013) Changes in student attitudes regarding science when taught by teachers without experiences with a model professional development program. School Science and Mathematics 113(3), 109-119.

Bandalos D.L., Finney S.J. (2010) Factor Analysis: Exploratory and Confirmatory. In G.R. Hancock and R.O. Mueller (Eds.), Reviewer's guide to quantitative methods. New York: Routledge.

Barak M., Ashkar T., Dori Y.J. (2011) Learning science via animated movies: Its effect on students' thinking and motivation. Computers \& Education 56(3), 839-846.

Blalock C., Lichtenstein M., Owen S., Pruski L., Marshall C., Toepperwein M. (2008) In pursuit of validity: A comprehensive review of science attitude instruments 19352005. International Journal of Science Education 30(7), 961-977.

Borsa J.C., Damásio B.F., Bandeira D.R. (2012) Cross-Cultural Adaptation and Validation of Psychological Instruments: some considerations. Paidéia 22(53), 423-432.

Brown T.A. (2006) Confirmatory factor analysis for applied research. New York: Guilford Press.

Caleon I.S., Subramaniam R. (2008) Attitudes towards science of intellectually gifted and mainstream upper primary students in Singapore. Journal of Research in Science Teaching 45(8), 940-954.

Cattell R.B. (1966) The scree test for the number of factors. Multivariate Behavioral Research 1, 245-276. 
Chi S., Wang Z., Liu X., Zhu L. (2017) Associations among attitudes, perceived difficulty of learning science, gender, parents' occupation and students' scientific competencies. International Journal of Science Education 39(16), 2171-2188.

Denessen E., Vos N., Hasselman F., Louws M. (2015) The Relationship between Primary School Teacher and Student Attitudes towards Science and Technology. Education Research International 2015, 1-7.

DeVellis R.F. (2003) Scale Development. Theory and Applications (2nd ed.). California: Sage Publications.

DeWitt J., Archer L. (2015) Who Aspires to a Science Career? A comparison of survey responses from primary and secondary school students. International Journal of Science Education 37(13), 2170-2192.

Ferrando P.J., Anguiano-Carrasco C. (2010) El análisis factorial como técnica de investigación en psicología. Papeles del Psicólogo 31(1), 18-33.

Fishbein M., Ajzen I. (1975) Belief, attitude, intention, and behavior: An introduction to theory and research. Reading, MA: Addison-Wesley.

Forero C.G, Maydeu-Olivares A., Gallardo-Pujol, D. (2009) Factor analysis with ordinal indicators: A monte Carlo study comparing DWLS and ULS estimation. Structural Equation Modeling 16, 625-641.

Fraser B.L. (1978) Development of a test of science-related attitudes. Science Education 62(4), 509-515.

Glynn S.M., Koballa T.R.Jr. (2006) Motivation to learn college science. In J.J. Mintzes, W.H. Leonard (Eds.), Handbook of college science teaching (pp. 25-32). Arlington: National Science Teachers Association Press.

Hair J.F., Black W.C., Babib B.J., Anderson R.E. (2010) Multivariate data analysis (7thed.). Upper Saddke River: Prentice Hall.

Jenkins E., Nelson N.W. (2005) Important but not for me: Students attitudes toward secondary school science in England. Research in Science \& Technological Education 23(1), 41-57.

Jung S. (2013) Exploratory factor analysis with small sample sizes: A comparison of three approaches. Behavioral Processes 97, 90-95.

Kennedy J., Quinn F., Taylor N. (2016) The school science attitude survey: A new instrument for measuring attitudes towards school science. International Journal of Research \& Method in Education 39(4), 422-445.

Kline R.B. (2005) Principles and practice of structural equation modeling (2nd Ed.). New York: Guilford.

Klopfer L. (1971) Evaluation of learning in science. In B.S. Bloom, J.T. Hastings and G.F. Madaus (Eds.), Handbook on summative and formative evaluation of student learning (pp. 559642). New York: McGraw-Hill.

Koballa T.R.Jr. (1988a) Attitude and related concepts in science education. Science Education 72(2), 115-126.

Koballa T.R.Jr. (1988b) The determinants of female junior high school students' intentions to enroll in elective physical science courses in high school: Testing the applicability of the theory of reasoned action. Journal of Research in Science Teaching 25(6), 479-492. 
Koballa T.R.Jr., Glynn S.M. (2007) Attitudinal and Motivational Constructs in Science Learning. In S.K. Abell, N.G. Lederman (Eds.), Handbook of Research on Science Education (pp. 75-102). Mahwah: Lawrence Erlbaum.

Lloret-Segura S., Ferreres-Traver A., Hernández-Baeza A., Tomás-Marco I. (2014) El análisis factorial exploratorio de los ítems: una guía práctica, revisada y actualizada. Anales de Psicología 30(3), 1151-1169.

Marbá-Tallada A., Márquez C. (2010) ¿Qué opinan los estudiantes de las clases de ciencias? Un estudio transversal de sexto de Primaria a cuarto de ESO. Enseñanza de las Ciencias 28(1), 19-30.

Mazas B., Bravo-Torija B. (2018) Actitudes hacia la ciencia del profesorado en formación de educación infantil y educación primaria. Profesorado. Revista de Currículum y Formación del Profesorado 22(2), 329-348.

Messick S. (1989) Meaning and values in test validation: The science and ethics of assessment. Educational Researcher 18(2), 5-11.

Ministerio de Educación, Cultura y Deporte (MECD) (2014a) Real Decreto 126/2014, de 28 de febrero, por el que se establece el currículo básico de la Educación Primaria.

Ministerio de Educación, Cultura y Deporte (MECD) (2014b) Real Decreto 1105/2014, de 26 de diciembre, por el que se establece el currículo básico de la Educación Secundaria Obligatoria y del Bachillerato.

Navarro M, Förster C, González C, González-Pose P. (2016) Attitudes toward science: Measurement and psychometric properties of the Test of Science-Related Attitudes for its use in Spanish-speaking classrooms. International Journal Science Education 38, 1459-1482

Newell A.D., Tharp B.Z., Moreno N.P., Zientek L.R., Vogt G.L. (2015) Students' attitudes toward science as predictors of gains on student content knowledge: Benefits of an after-school program. School Science and Mathematics 115, 216-225.

Osborne J., Simon S., Collins, S. (2003) Attitudes towards science: a review of the literature and its implications. International Journal of Science Education 25(9), 1049-1079.

Pearl R.E. (1974) The present status of science attitude measurement: History, theory, and availability of measurement instruments. School Science and Mathematics 74(5), 375-381.

Pell T., Jarvis T. (2001) Developing attitude to science scales for use with children of ages from five to eleven years. International Journal in Science Education 23(8), 847-862.

Pérez-Manzano A. (2012) Actitudes hacia la ciencia en primaria y secundaria (Tesis Doctoral). España: Universidad de Murcia.

Potvin P., Hasni, A. (2014) Interest, motivation and attitude towards science and technology at K-12 levels: a systematic review of 12 years of educational research. Studies in Science Education 50(1), 85-129.

Pro A. de, Pérez-Manzano A. (2014) Primary and Secondary students' attitude towards the dichotomic view of Science. Enseñanza de las Ciencias 32(3), 111-132.

Ramsden J.M. (1998) Mission impossible? Can anything be done about attitudes to science? International Journal of Science Education 20(2), 125-137.

Richardson V. (1996) The role of attitudes and beliefs in learning to teach. In J. Sikula (Ed.), Handbook of research on teacher education (pp. 102-119). New York: MacMillan. 
Rodríguez-Menéndez M.C., Peña-Calvo J.V., Inda-Caro M.M. (2016) "Esto es lo que me gusta y lo que voy a estudiar": Un estudio cualitativo sobre la toma de decisiones académicas en bachillerato. Revista Complutense de Educación 27(3), 1351-1368.

Said Z., Summers R., Abd-El-Khalick F., Wang S. (2016) Attitudes toward science among grades 3 through 12 Arab students in Qatar: findings from a cross-sectional national study. International Journal of Science Education 38(4), 621-643.

Schermelleh-Engel K., Moosbrugger H., Müller H. (2003) Evaluating the fit of structural equation models: Tests of significance and descriptive goodness-of-fit measures. Methods of Psychological Research Online 8(2), 23-74.

Schwarz N. (2007) Attitude construction: evaluation in context. Social Cognition 25(5), 638-656.

Sjøberg S., Schreiner C. (2010) The ROSE project. An overview and key findings. Recuperado de: http://roseproject.no/network/countries/norway/eng/nor-Sjoberg-Schreineroverview-2010.pdf

Sjøberg S., Schreiner, C. (2005) How do learners in different cultures relate to science and technology? Asia-Pacific Forum on Science Learning and Teaching 6(2), 1.

Speering W., Rennie L. (1996) Students' perceptions about science: The impact of transition from primary to secondary school. Research in Science Education 26(3), 283-298.

Stark R., Gray D. (1999) Gender preferences in learning science. International Journal of Science Education 21(6), 633-643.

Summers R., Abd-El-Khalick, F. (2018) Development and validation of an instrument to assess student attitudes toward science across grades 5 through 10. Journal of Research in Science Teaching 55(2), 172-205.

Toma R.B., Greca I.M. (2018) The Effect of Integrative STEM Instruction on Elementary Students' Attitudes toward Science. Eurasia Journal of Mathematics, Science and Technology Education 14(4), 1383-1395.

Toma R.B., Meneses-Villagrá J.A. (2019) Validation of the single-items Spanish-School Science Attitude Survey (S-SSAS) for elementary education. PLOS ONE 14(1), e0209027.

Tytler R. (2014) Attitudes, identity, and aspirations toward science. In S. Abell \& N. Lederman (Eds.), Handbook of research on science education volume II (pp. 82-103). New York: Routledge.

Ullman J.B. (2006) Structural equation modeling. In B.G. Tabachnick, L.S. Fidell (Eds.). Using multivariate statistics (5th ed.) (pp. 653-771). Boston: Allyn \& Bacon.

Usher E.L., Pajares F. (2009) Sources of self-efficacy in mathematics: a validation study. Contemporary Educational Psychology 34, 89-101.

Vázquez A., Manassero M. A. (1997). Una evaluación de las actitudes relacionadas con la ciencia. Enseñanza las Ciencias 15(2), 199-213.

Vázquez A., Manassero M. A. (2008) El declive de las actitudes hacia la ciencia de los estudiantes: un indicador inquietante para la educación científica. Revista Eureka sobre Enseñanza y Divulgación de las Ciencias 5(3), 274-292.

Vedder-Weis D., Fortus D. (2011) Adolescents' Declining Motivation to Learn Science: Inevitable or Not? Journal of Research in Science Teaching 48(2), 199-216. 


\section{Anexo I: Cuestionario SSAQ}

Se presenta en español e inglés. La versión inglesa ha sido adaptada de Barak et al. (2011).

1. Pienso que la ciencia es un tema interesante. I think that science is an interesting subject.

2. En clase de ciencias obtengo respuestas a preguntas que me intrigan. In science lessons I can get answer to questions that intrigue me.

3. En clase de ciencias puedo expresar mis propias ideas. In science lessons I can express my own ideas.

4. Puedo tener éxito en la ciencia sin la ayuda del profesor. I can succeed in science even without the teacher's help.

5. Las asignaturas de ciencias no deberían ser obligatorias en la escuela. Science shouldn't be an obligatory subject in schools.

6. El número de horas por semana de ciencias debería incrementarse. The number of hours per-week for science lessons should be increased.

7. Las clases de ciencias me fascinan. Science lessons fascinate me.

8. Las clases de ciencias me aburren. Science lessons bore me.

9. Es importante para mí entender lo que se enseña en clase de ciencias. It is important for me to understand the topics taught in science lessons.

10. Las lecciones de ciencias son fáciles de estudiar. Science lessons are easy for me to study.

11. Me divierto aprendiendo ciencias. I enjoy learning science.

12. En el futuro me gustaría ser científico. In the future I would like to be a scientist.

13. Los estudios científicos me permiten entender fenómenos cotidianos. Science studies enable me to understand daily phenomenon.

14. Confío en mis habilidades para tener éxito en el estudio de las ciencias. I have confidence in my ability to succeed in science studies.

15. Ayudo a los demás en las clases de ciencias. I help others in science lessons.

16. Me gusta leer artículos y ver programas que tratan temas científicos. I like to read articles and watch TV broadcasts that present science topics.

17. Me interesan las explicaciones de los fenómenos científicos. I am interested in explanations of scientific phenomenon.

18. Creo que la comprensión de la ciencia es importante para todos. I think that understanding science is important to everyone.

19. Es difícil para mí aprender ciencia. It is difficult for me to learn science.

20. La ciencia no tiene conexión con mi vida. Science has no connection to my life. 